\title{
Phase II studies of polymer-doxorubicin (PK1, FCE28068) in the treatment of breast, lung and colorectal cancer
}

\author{
LEONARD W. SEYMOUR ${ }^{1,10}$, DAVID R. FERRY ${ }^{1,11}$, DAVID J. KERR ${ }^{1,10}$, DANIEL REA ${ }^{1}$, MAGGIE WHITLOCK ${ }^{1}$, \\ RICHARD POYNER $^{2}$, CHRISTOPHER BOIVIN ${ }^{2}$, STUART HESSLEWOOD ${ }^{3}$, CHRISTOPHER TWELVES ${ }^{4,12}$, \\ ROBERT BLACKIE ${ }^{4}$, ANDREAS SCHATZLEIN ${ }^{4,13}$, DUNCAN JODRELL ${ }^{5}$, DONALD BISSETT ${ }^{6}$, \\ HILARY CALVERT ${ }^{7}$, MIKE LIND $^{14}$, ADELE ROBBINS $^{8}$, SALLY BURTLES $^{8}$, \\ RUTH DUNCAN $^{9}$ and JAMES CASSIDY ${ }^{6,15}$
}

\begin{abstract}
${ }^{1}$ Cancer Research UK Institute for Cancer Studies, University of Birmingham, Birmingham B15 2TT;
${ }^{2}$ Department of Nuclear Medicine, Queen Elizabeth Hospital, University Hospital Birmingham NHS Trust, Birmingham B15 2TH; ${ }^{3}$ Department of Physics and Nuclear Medicine, City Hospital NHS Trust, Dudley Road, Birmingham B18 7QH; ${ }^{4}$ Department of Oncology, Cancer Research UK Beatson Laboratories, University of Glasgow, Garscube Estate, Bearsden, Glasgow G61 1BP; ${ }^{5}$ Cancer Research UK, Edinburgh Oncology Unit, Western General Hospital, Edinburgh EH4 2XR;

${ }^{6}$ Department of Medical Oncology, University of Aberdeen, Institute of Medical Science, Foresterhill, Aberdeen AB25 2ZD; ${ }^{7}$ Northern Institute for Cancer Research, University of Newcastle, Newcastle NE2 4HH;

${ }^{8}$ Drug Development Office, Cancer Research UK, 61 Lincoln's Inn Fields, London WC2A 3PX;

${ }^{9}$ School of Pharmacy, King Edward VII Avenue, Cardiff, UK
\end{abstract}

Received January 12, 2009; Accepted February 24, 2009

DOI: 10.3892/ijo_00000293

\begin{abstract}
Phase I studies of [N-(2-hydroxypropyl)methacrylamide] (HPMA) copolymer-doxorubicin previously showed signs of activity coupled with 5-fold decreased anthracycline toxicity in chemotherapy-refractory patients. Here we report phase II studies using a similar material (FCE28068) in patients with breast $(n=17)$, non-small cell lung (NSCLC, $n=29)$ and colorectal $(n=16)$ cancer. Up to 8 courses of PK1 $\left(280 \mathrm{mg} / \mathrm{m}^{2}\right.$ doxorubicin-equivalent) were given i.v., together with ${ }^{123} \mathrm{I}-$ labelled imaging analogue. Toxicities were tolerable, with grade 3 neutropenia more prominent in patients with breast cancer $(4 / 17,23.5 \%$ compared with $5 / 62,8.1 \%$ overall). Of
\end{abstract}

Correspondence to: Professor L.W. Seymour, Department of Clinical Pharmacology, University of Oxford, Old Road Campus Research Building, Headington, Oxford OX3 7DQ, UK

E-mail: len.seymour@clinpharm.ox.ac.uk

Present addresses: ${ }^{10}$ Department of Clinical Pharmacology, University of Oxford, Oxford OX3 7DQ; ${ }^{11}$ Department of Oncology, New Cross Hospital, Wolverhampton WV10 0QP; ${ }^{12}$ Cancer Research UK Clinical Centre in Leeds, St James University Hospital, Leeds LS9 7TF; ${ }^{13}$ School of Pharmacy, University of London, Brunswick Square, London WC1N 1AX; ${ }^{14}$ Department of Academic Oncology, Princess Royal Hospital, Hull HU8 9HE; ${ }^{15}$ Department of Oncology, Cancer Research UK Beatson Laboratories, University of Glasgow, Garscube Estate, Bearsden, Glasgow G61 1BP, UK

Key words: drug targeting EPR effect, [N-(2-hydroxypropyl)methacrylamide] copolymer conjugates, polymeric produgs
14 evaluable patients with breast cancer 3 had partial responses (PR), all anthracycline-naïve patients. In 26 evaluable patients with NSCLC, 3 chemotherapy-naïve patients had PR. In contrast, none of the 16 evaluable patients with colorectal cancer responded. Imaging of 16 patients ( 5 with breast cancer, 6 NSCLC, 5 colorectal cancer) showed obvious tumour accumulation in 2 metastatic breast cancers, although unfortunately no images were obtained from patients who responded. These results show 6/62 PR with limited side effects, supporting the concept that polymer-bound therapeutics can have modified and improved anticancer activities and suggesting the approach should be explored further for breast cancer and NSCLC.

\section{Introduction}

Targeting drugs to sites of disease is a long-held aim in pharmacology, promising to combine improved activity with decreased exposure of non-target tissues (1). This goal is particularly important for cancer chemotherapy, where dose is often limited by toxicities in normal tissues, notably proliferating cells such as those of the intestinal mucosa and bone marrow. One means to achieve drug targeting in cancer involves linking the cytotoxic to high molecular weight carrier molecules, such as hydrophilic polymers (2-4). This affords the possibility to attach specific ligands to the polymeric carrier, to achieve 'active targeting' to cancer cell-associated receptors. The modified pharmacokinetic properties of the polymer-drug conjugate have also been found to mediate selective accumulation within model tumours, so called 'passive targeting' (5). The mechanism of passive tumour targeting, otherwise known as the enhanced permeability and 
retention (EPR) effect, is thought to result from increased permeability of tumour vasculature, enabling extravasation of high molecular weight molecules such as proteins and polymerdrug conjugates from the bloodstream, coupled with disorganised drainage of tissue fluid from tumour interstitium leading to retention of macromolecules $(6,7)$. EPR thus leads to high levels of passive targeting of polymer-drug conjugates in animal model tumours (8), although it is still unclear how important is the EPR effect in clinical disease, where tumour growth can be considerably slower than in animal models.

The first soluble polymer-drug for cancer therapy was evaluated in a phase I trial against solid tumours in the UK $(9,10)$. This agent, FCE28068 (known as 'PK1'), consists of the anthracycline doxorubicin linked to copolymers based on $\mathrm{N}$-(2-hydroxypropyl)methacrylamide via a tetrapeptide spacer designed for cleavage by lysosomal cathepsins (11). Following endocytic entry into tumour cells the spacer is cleaved, allowing intracellular release of active doxorubicin $(3,12,13)$. In the phase I trial, FCE28068 was found to mediate $\sim 5$-fold decreased toxicity than the equivalent dose of free doxorubicin, with the maximum tolerated dose identified as $320 \mathrm{mg} / \mathrm{m}^{2}$. Nine patients were evaluated for effects on cardiac function, receiving a mean cumulative dose of FCE28068 (doxorubicinequivalent) of $782 \mathrm{mg} / \mathrm{m}^{2}$. One of these showed a clinical significant fall in LVEF (from $47 \%$ to $30 \%$; cumulative doxorubicin-equivalent dose of $1040 \mathrm{mg} / \mathrm{m}^{2}$ ), although none of the others showed any signs of qualitative or quantitative changes in cardiac function. The dose limiting toxicity was neutropaenia. Tolerability was confirmed in a further 6 patients treated at $280 \mathrm{mg} / \mathrm{m}^{2}$ (doxorubicin-equivalent), the dose recommended for further trials.

Results of the phase I trial were encouraging, with two patients with NSCLC achieving complete responses (CR) in individual lesions and partial responses (PR) overall (overall duration of PR 10.6 and 16.7 weeks) (9). There were also two minor responses, including one patient with primary colorectal cancer showing ultrasound-documented partial response of a measurable hepatic metastasis with other lesions stable, and the other reduction of chest wall lesions in a patient with heavily epirubicin-pretreated anthracyclinerefractory breast cancer. Of the 36 patients enrolled on the phase I trial, 21 were imaged with ${ }^{131}$ I-labelled polymer conjugate and 6/21 showed some evidence for accumulation of the labelled conjugate within tumour lesions. Accurate determination of the level of tumour accumulation was difficult, however, as ${ }^{131} \mathrm{I} \gamma$-camera imaging does not allow precise resolution of tumour-associated radioactivity.

Given the preliminary evidence of therapeutic activity in NSCLC and some indications of non-specific tumour accumulation, together with decreased toxicity of the HPMA copolymer-bound doxorubicin and lack of polymer-related toxicity, the Phase I/II Clinical Trials Committee of the Cancer Research Campaign approved PK1 for further evaluation in three multicentre phase II trials. Target diseases were breast cancer, NSCLC and colorectal cancer. Drug distribution was monitored in a subset of patients by $\gamma$-camera imaging using a ${ }^{123}$ I-labelled HPMA copolymer-doxorubicin conjugate, to confirm biodistribution, assess uptake into solid tumours and enable assessment of pharmacokinetics by monitoring radioactivity in samples of blood and urine.

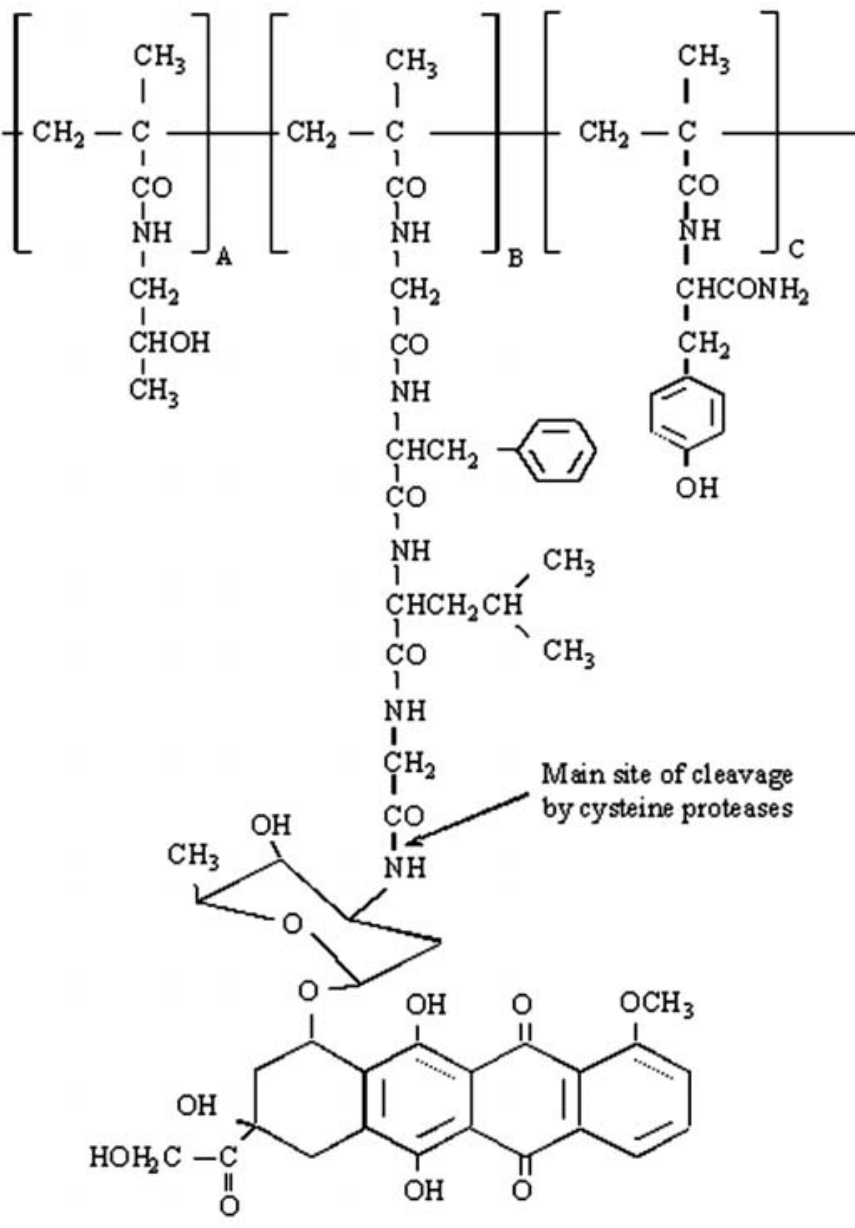

Figure 1. General chemical structures of doxorubicin polymer conjugates employed. FCE28068 is a copolymer of (2-hydroxypropyl)methacrylamide bearing doxorubicin, with $\mathrm{A}=96.1, \mathrm{~B}=3.9 \mathrm{~mol} \%$. An imaging analogue of FCE28068 was also prepared to permit radio-iodination, containing additionally $\mathrm{C}=1.0$, with $\mathrm{A}=95 \mathrm{~mol} \%$ approximately.

\section{Patients and methods}

FCE28068 was supplied for these trials by Pharmacia and Upjohn as a lyophilized powder, in glass vial containing $50 \mathrm{mg}$ doxorubicin-equivalent. This drug conjugate has weight average molecular weight 29000 and contains $12 \%$ doxorubicin-equivalent, by weight $(3.9 \mathrm{~mol} \%)$. An imaging analogue was also supplied by Pharmacia and Upjohn, which contained additionally methacryloyltyrosinamide (1 mol \%) to permit radio-iodination (Fig. 1). This imaging analogue was iodinated with ${ }^{123} \mathrm{I}$-iodide in the presence of carrier iodide, using a protocol described elsewhere (14). The drug was purified by gel permeation chromatography (Sephadex G25) immediately prior to intravenous administration.

Patients and clinical protocol. This study was conducted in five centers throughout the UK. Patients with histologically- or clinically-confirmed breast carcinoma (enrolled in Newcastle, Glasgow and Birmingham), non-small cell lung cancer (enrolled in Birmingham and Aberdeen) or colorectal carcinoma (enrolled in Aberdeen, Edinburgh and Birmingham) were eligible. All imaging studies were performed in Birmingham. The studies were reviewed and approved by 
the Local Research Ethics Committees and ARSAC (Administration of Radioactive Substances Advisory Committee), and all patients gave written informed consent. Toxicity was documented using the Expanded CTC of the Clinical Trials Group of the National Cancer Institute of Canada version 1 (NCIC-CTG ECTC), and responses were recorded according to criteria based on standard WHO criteria. Inclusion criteria for all three trials included the presence of metastatic disease, at least one bidimensionally measurable lesion, serum creatinine $\leq 150 \mu \mathrm{mol} / 1$, serum bilirubin $\leq 20 \mu \mathrm{mol} / 1$, serum liver enzymes $\leq 2 \mathrm{X}$ upper limit of the normal range (ULN), except for patients with liver metastases $\leq 5 \mathrm{X}$ ULN and life expectancy at least 3 months. Exclusion criteria included a prior cumulative dose of epirubicin $>450 \mathrm{mg} / \mathrm{m}^{2}$ or doxorubicin $>200 \mathrm{mg} / \mathrm{m}^{2}$, pregnant or lactating females, unresolved toxicity, clinical evidence of brain disease, poor medical risk, concurrent use of other experimental agents, treatment for congestive heart failure and history of other malignancies (except in situ cervical carcinoma or basal/squamous cell skin carcinoma curatively treated).

\section{Trial-specific enrollment criteria included:}

Breast cancer patients. Patients were required to have received at least one line of previous chemotherapy. Patients who had received prior anthracyclines were required to have a left ventricular ejection fraction (LVEF) measured by multigated radionuclide angiography (MUGA) or echocardiography (ECHO) within the normal range for each hospital and the LVEF measurement was repeated after every 2 cycles of FCE28068. A protocol amendment partway through the trial changed the prior-anthracycline limits to $>450 \mathrm{mg} / \mathrm{m}^{2}$ (epirubicin) and $>240 \mathrm{mg} / \mathrm{m}^{2}$ (doxorubicin).

Lung cancer patients. Patients progressing on cisplatin chemotherapy or with progressive disease less than 6 months from completing such treatment were excluded. Most patients had not received any prior chemotherapy.

Patients were treated with FCE28068 at a fixed dose of $280 \mathrm{mg} / \mathrm{m}^{2}$ (doxorubicin-equivalent) by short intravenous infusion of a $2-\mathrm{mg} / \mathrm{ml}$ (doxorubicin-equivalent) solution in normal saline, at a rate of $4 \mathrm{ml} / \mathrm{min}$ and repeated every 3 weeks. For imaging cycles, patients were subject to thyroid blockade with potassium perchlorate. FCE28068 and ${ }^{123}$ I-labelled imaging analogue (for imaging cycles only) were administered simultaneously in $0.9 \%$ saline by intravenous infusion. Treatment was to be continued for a maximum of 6 cycles. For analysis of pharmacokinetics, blood samples were taken into heparinized tubes immediately before treatment, and at 15 and $30 \mathrm{~min}$ after the start of infusion, then at the end of infusion (to give peak drug level) and at 5, 10, 15, 30, $60 \mathrm{~min}$, $2,4,8,24 \mathrm{~h}$ and 7 days after the end of infusion. Samples were immediately centrifuged, and plasma isolated for measurement of contained radioactivity. Urine was collected in three samples (0-4 h, 4-12 h, 12-24 h).

Analysis of plasma and urine samples. Levels of ${ }^{123} \mathrm{I}$-iodide radioactivity were measured in samples of plasma and urine, diluted to standard volume, by $\gamma$-scintigraphy in a well counter (Cobra Quantum, Canberra Packard) and calibrated against a known dilution of imaging analogue. Urine samples were fractionated by Sephadex G25 chromatography before analysis of the fractions to determine the component of free iodide.

Imaging procedures for determination of polymer distribution. Some patients at the Birmingham trial site were given a small amount of ${ }^{123} \mathrm{I}$-imaging analogue as a tracer, administered simultaneously with unlabelled FCE28068, during one of their therapeutic cycles. Planar anterior and posterior whole body $\gamma$-camera images were taken 4,24 and $48 \mathrm{~h}$ after infusion, and SPECT imaging was performed at $24 \mathrm{~h} \mathrm{(15).} \mathrm{A}$ standard of ${ }^{123}$ I-labelled imaging analogue was included by the feet of the patient in the planar images to aid in absolute quantification and correction for time-dependent radioactive decay. All imaging was performed using an ADAC Vertex EPIC 2 dual-headed $\gamma$-camera. Quantification of ${ }^{123}$ I distribution was performed from the whole body scans using the geometric mean with comparison to a ${ }^{123}$ I-iodide standard measured through the patient before the infusion commenced, to enable correction for signal attenuation. The day before treatment the patient underwent an i.v. and oral contrast enhanced X-ray CT scan (GE Prospeed) with markers placed over exterior anatomical landmarks. This was performed at mid-inspiration (rather than full breath-hold) to give a more representative image for comparison to the SPECT scan (which was performed with quiet respiration). A brief SPECT scan was performed with markers in the same locations as for CT before full SPECT imaging limited to the area of ${ }^{123} \mathrm{I}$ distribution to permit registration of the tomographic scans. SPECT to CT registration was performed with both modalities transferred to a Hermes workstation (Nuclear Diagnostics, Stockholm, Sweden), using the Multimodality application based on the external markers, described above, with the kidneys being employed as additional, internal, markers.

\section{Results}

Patients. Sixty-two patients were enrolled into the study, with breast $(\mathrm{n}=17)$, non-small cell lung $(\mathrm{n}=29)$ and colorectal $(n=16)$ carcinoma; 32 were male and 30 female (Table I). The diagnosis of malignancy was confirmed histologically or cytologically with the exception of 9 patients diagnosed with lung cancer on clinical and radiological grounds.

\section{Anti-tumour activity}

Breast cancer. Seventeen patients with metastatic breast cancer were treated with FCE28068, of whom 14 were evaluable for tumour responses. The other three patients were too ill to have complete assessment, of whom two had symptomatic progression. Two evaluable patients showed early disease progression, four had progressive disease (PD), five had stable disease (SD), and three had PR. The three PR were all observed in patients who had not received prior anthracycline therapy, although they had all previously received radiotherapy and been treated with cyclophosphamide, fluorouracil and methotrexate. The first breast cancer patient achieving PR had two lung metastases, one of which disappeared and the other achieved a PR. This patent also had lesions in her chest wall that were initially measurable but became non-evaluable on study (Fig. 2). The response was maintained for 24 months, when the patient relapsed with pleural effusion. 
Table I. Summary of patient characteristics and treatments.

\begin{tabular}{lcccc}
\hline Primary disease & $\begin{array}{c}\text { No. of patients } \\
(\mathrm{M}: \mathrm{F})\end{array}$ & $\begin{array}{c}\text { No. of FCE28068 } \\
\text { cycles administered }\end{array}$ & $\begin{array}{c}\text { Age range } \\
\text { (years) }\end{array}$ & $\begin{array}{c}\text { No. of prior regimens } \\
\text { of chemotherapy }\end{array}$ \\
\hline Breast & $0: 17$ & 66 & $34-73$ & Range 1-3 (median 2) \\
Colorectal & $12: 4$ & 53 & $45-76$ & Range 1-2 (median 1) \\
Lung & $20: 9$ & 91 & $36-77$ & Range 0-1 (median 0) \\
\hline
\end{tabular}
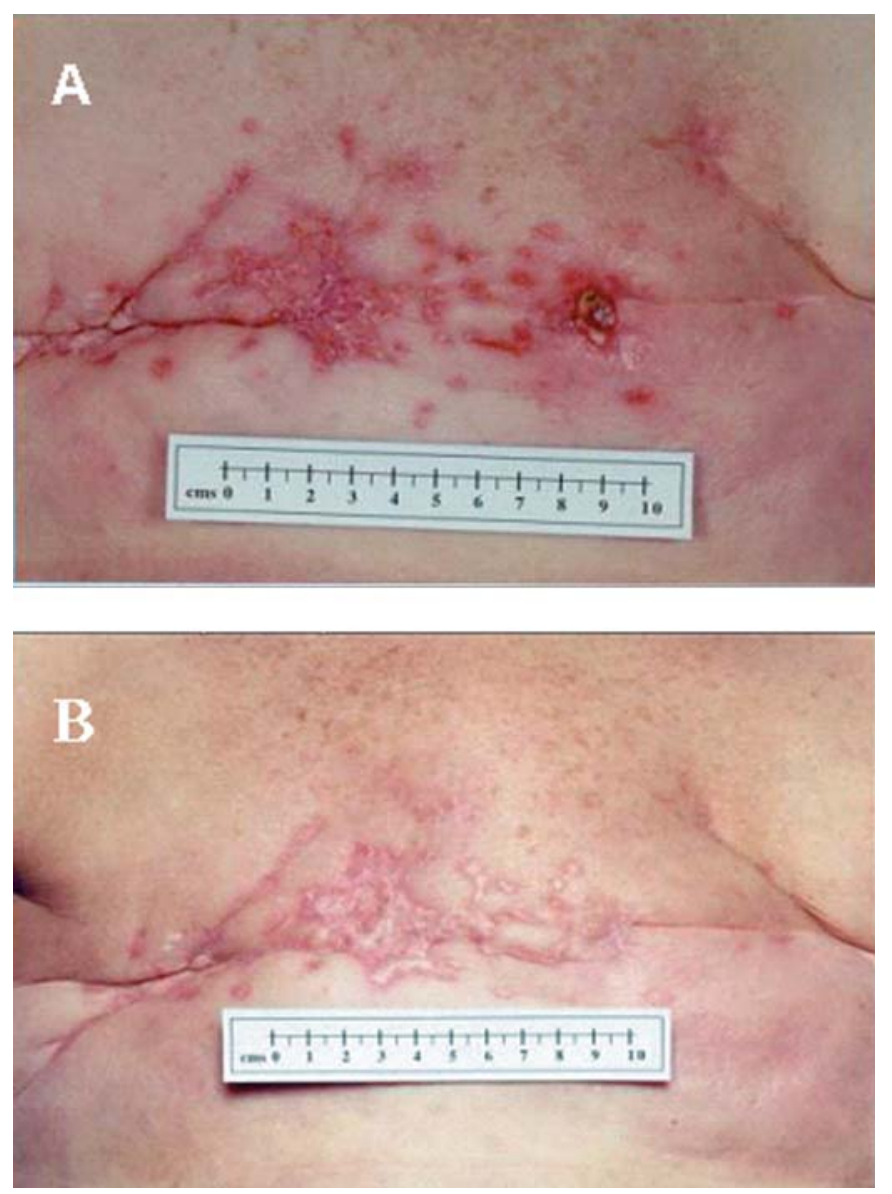

Figure 2. Chest wall lesions in a patient with breast cancer receiving FCE28068. Superficial lesions prior to treatment (A) became less well defined and showed signs of remission (B) during treatment.

The second breast cancer patient achieving PR had a liver metastasis that showed greater than $50 \%$ reduction by $\mathrm{CT}$ scan (bi-dimensional area measurement), with lung and bone metastases that were non-measurable but judged to be unchanged. This patient was in PR after six treatment cycles and received an additional two cycles.

The third breast cancer patient achieving overall PR had a mediastinal lymph node lesion that showed $>50 \%$ size reduction, confirmed by $\mathrm{CT}$ scan, before the patient decided to come off treatment after 5 cycles.

Non-small cell lung cancer. Twenty-nine patients with NSCLC were treated with FCE28068, of whom 26 completed two courses of treatment and were therefore evaluable for therapeutic response. Of these 26 patients treated, response was assessed objectively in 21 . Three patients showed early progression while seven had PD and eight SD; three patients, all chemotherapy naïve, achieved a PR. These comprised two patients who showed shrinkage of the primary lesion, one achieving greater than $50 \%$ reduction in bidimensional area, documented by planar X-ray, and the other achieving complete disappearance documented by CT scan. A third patient showed greater than $50 \%$ reduction of locally recurrent lung cancer, documented by X-ray. A fourth chemotherapy-naïve patient had a reduction in the primary tumour that appeared to meet the criteria for PR, but could not formally be classified as a responder because the follow-up X-ray images could not be directly compared with the baseline films.

Colorectal cancer. Sixteen patients with metastatic colorectal cancer were treated. Although 5 patients had stable disease, there were no responses or significant falls in serum CEA.

Assessment of toxicity. Drug-related toxicities observed were generally tolerable, and similar for the three disease groups (Table II). The dose-limiting toxicities observed in the phase I trial were neutropenia and stomatitis, and these were frequently observed in patients treated in this study. Patients with breast cancer experienced somewhat more grade 3 neutropenia (4/17) than those with colorectal $(1 / 16)$ or lung $(0 / 29)$ cancer. Grade 3 stomatitis was experienced by all patient groups $(3 / 17,3 / 16$ and $1 / 29$ patients with breast, colorectal and lung cancer, respectively). There were no episodes of grade 4 neutropenia or febrile neutropenia. Alopecia was limited (Table II), with only 3 colorectal and 3 NSCLC patients experiencing grade 3 hair loss. Other toxicities, including anemia and thrombocytopenia, were either minor or occurred infrequently. Finally, patients with breast cancer reported drug-related fatigue more frequently (13/17) than those with colorectal (7/16) or lung $(12 / 29)$ cancer. Fatigue is difficult to quantify, however, and this is thought to reflect slight variation in interpretation in the different centers. There was no sign of any cardiotoxicity.

Pharmacokinetics of FCE28068. Plasma and urine samples from the 16 patients subject to $\gamma$-camera imaging were analysed by $\gamma$-scintigraphy to determine the distribution of the polymer carrier. Plasma levels of radioactivity were assessed using a Winnonlin nonlinear estimation program and modeled well to bi-exponential kinetics, distribution half life $=3.0 \mathrm{~h}$ and elimination half life $=41.2 \mathrm{~h}$ (Fig. 3), with AUC (area under the plasma concentration-time curve, $0-\infty$ h) of $5.0 \mathrm{mM}$.h. Samples of urine were also analysed in 15 patients, with the 
Table II. Toxicities encountered following administration of FCE28068.

\begin{tabular}{|c|c|c|c|c|c|c|c|c|c|c|c|c|c|c|c|c|c|c|c|c|}
\hline \multirow{2}{*}{$\begin{array}{l}\text { Disease } \\
\text { No. of patients treated } \\
\text { CTC grade }\end{array}$} & \multicolumn{5}{|c|}{$\begin{array}{c}\text { Breast } \\
17\end{array}$} & \multicolumn{5}{|c|}{$\begin{array}{c}\text { Colorectal } \\
16\end{array}$} & \multicolumn{5}{|c|}{$\begin{array}{c}\text { Lung } \\
29\end{array}$} & \multicolumn{5}{|c|}{$\begin{array}{c}\text { Overall } \\
62\end{array}$} \\
\hline & 0 & 1 & 2 & 3 & 4 & 0 & 1 & 2 & 3 & 4 & 0 & 1 & 2 & 3 & 4 & 0 & 1 & 2 & 3 & 4 \\
\hline Alopecia & 8 & 5 & 4 & & & 9 & 6 & 1 & & & 22 & 3 & 1 & 3 & & 39 & 14 & 6 & 3 & \\
\hline Nausea & 7 & 8 & 2 & & & 10 & 3 & 2 & 1 & & 20 & 5 & 2 & 2 & & 37 & 16 & 6 & 3 & \\
\hline Vomiting & 15 & 1 & 1 & & & 14 & 1 & & 1 & & 26 & 1 & 1 & 1 & & 55 & 3 & 2 & 2 & \\
\hline Diarrhoea & 14 & 2 & 1 & & & 12 & 2 & & 2 & & 27 & 1 & 1 & & & 53 & 5 & 2 & 2 & \\
\hline Stomatitis & 8 & 4 & 2 & 3 & & 10 & 1 & 2 & 3 & & 17 & 8 & 3 & 1 & & 35 & 13 & 7 & 7 & \\
\hline Pain & 11 & 3 & 2 & 1 & & 13 & & 1 & 2 & & 26 & & 3 & & & 50 & 3 & 6 & 3 & \\
\hline Fatigue & 4 & 4 & 8 & 1 & & 9 & 2 & 3 & 2 & & 17 & 2 & 8 & 2 & & 30 & 8 & 19 & 5 & \\
\hline WBC & 5 & 3 & 5 & 4 & & 10 & 2 & 2 & 2 & & 16 & 5 & 5 & 3 & & 31 & 10 & 12 & 9 & \\
\hline Neutropenia & 5 & 3 & 5 & 4 & & 11 & 2 & 2 & 1 & & 18 & 5 & 6 & & & 34 & 10 & 13 & 5 & \\
\hline Lymphocytopenia & 6 & & 3 & 4 & 4 & 4 & 1 & 3 & 6 & 2 & 12 & 3 & 9 & 5 & & 22 & 4 & 15 & 15 & 6 \\
\hline
\end{tabular}

Toxicities shown are those reported more than once at grade 3 and above. Other specific toxicities, encountered once only at grade 3, were: infection, anorexia, neuro-headache, neuro-constipation, hot flushes and potassium deficiency.

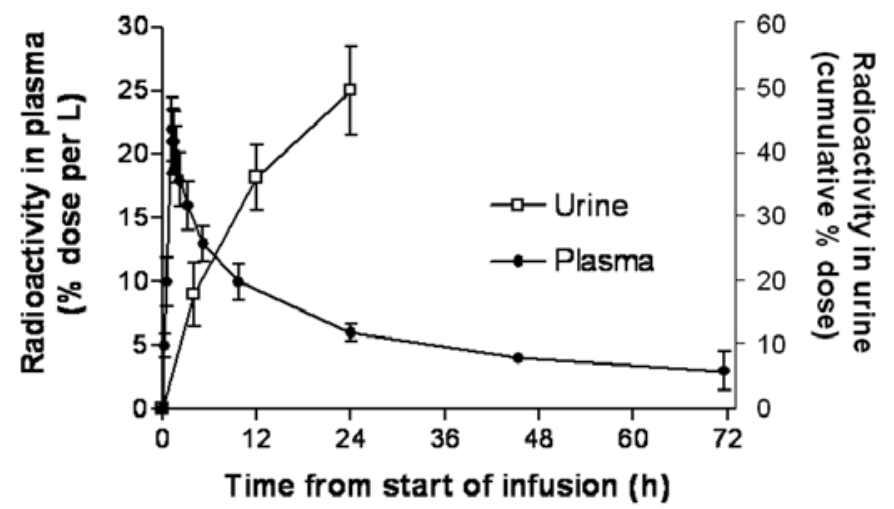

Figure 3. Average plasma (circles) and urine (squares) levels of FCE28068 determined from analysis of radioactivity in samples from imaged patients following intravenous injection at a doxorubicin-equivalent dose of $280 \mathrm{mg} / \mathrm{m}^{2}$. A trace dose of the ${ }^{123}$ I-labelled imaging analogues was premixed with the unlabelled FCE28068 and levels of ${ }^{123}$ I were measured by $\gamma$-scintigraphy. Plasma data are expressed as percentage dose administered per L, and urine data are cumulative percentage administered dose excreted. Mean values are shown, with error bars representing \pm SD when 3 samples or more were taken (from different patients) at the same time.

overall percentage radioactivity excreted during $24 \mathrm{~h}$ found to be $50.3 \pm 7.0 \%$ (mean $\pm 95 \%$ confidence interval).

$\gamma$-camera imaging of the biodistribution of ${ }^{123}$ I-imaging analogue of FCE28068. A total of 16 patients (five with breast cancer, five with colorectal cancer and six with lung cancer) underwent whole body $\gamma$-camera imaging 4, 24 and $48 \mathrm{~h}$ following administration of ${ }^{123} \mathrm{I}$-labelled imaging analogue of FCE28068. In the majority of patients, images were similar to that in Fig. 4A, with the radioactivity still concentrated in the bloodstream after $4 \mathrm{~h}$, the heart and major vessels being clearly visible. There was also significant radioactivity in the kidneys and bladder, reflecting urinary excretion of the drug conjugate. After $24 \mathrm{~h}$ there was still evidence of radioactivity in the bloodstream, although the whole body level had fallen considerably, approximately $50 \%$ of radioactivity being excreted in the first $24 \mathrm{~h}$. By registering the $\gamma$-camera images to the SPECT, levels of radioactivity in the liver at $24 \mathrm{~h}$ were calculated as $1.9 \pm 0.9 \%$ of the injected dose. Slight accumulation in the kidneys was noted $(5.8 \pm 2.5 \%$ of the injected dose), possibly reflecting post-glomerular reabsorption of the polymer into the renal tubular epithelium. In some breast cancer patients tumour deposits could be imaged by SPECT, although no evidence was gained for enhanced tumour uptake of radioactivity in patients with colorectal and lung cancer.

Two patients with breast cancer had tumour masses arising from axillary lymph node metastases. Radioimaging in these patients showed uptake into the tumour mass. This accounted for $5.9 \%$ and $1.8 \%$ of the injected dose after $24 \mathrm{~h}$ with some retention over the $48 \mathrm{~h}$ period of study, compared with matched uptake into the contra-lateral area of $2.9 \%$ and $1.1 \%$, respectively. From the $\gamma$-camera image of the patient with $5.9 \%$ accumulation in the left axillary it is clear that the tumour represents one of the dominant sites of radioactivity remaining within the body after $48 \mathrm{~h}$ (Fig. 4B).

\section{Discussion}

These studies have expanded and confirmed the early indications that FCE28068 has therapeutic activity against some advanced solid neoplasms. In patients with metastatic breast and lung cancer, FCE28068 achieved response rates of $21 \%$ and $11.5 \%$, respectively; there was, however, no activity against colorectal cancer. The activity in breast cancer was restricted to anthracycline-naïve patients, and it is possible that free doxorubicin could also have been effective in these patients. The activity of FCE28068 against NSCLC is particularly encouraging, achieving response rates of $2 / 2$ and $3 / 26$ (on an intent to treat basis) in the phase I and II trials, 
A

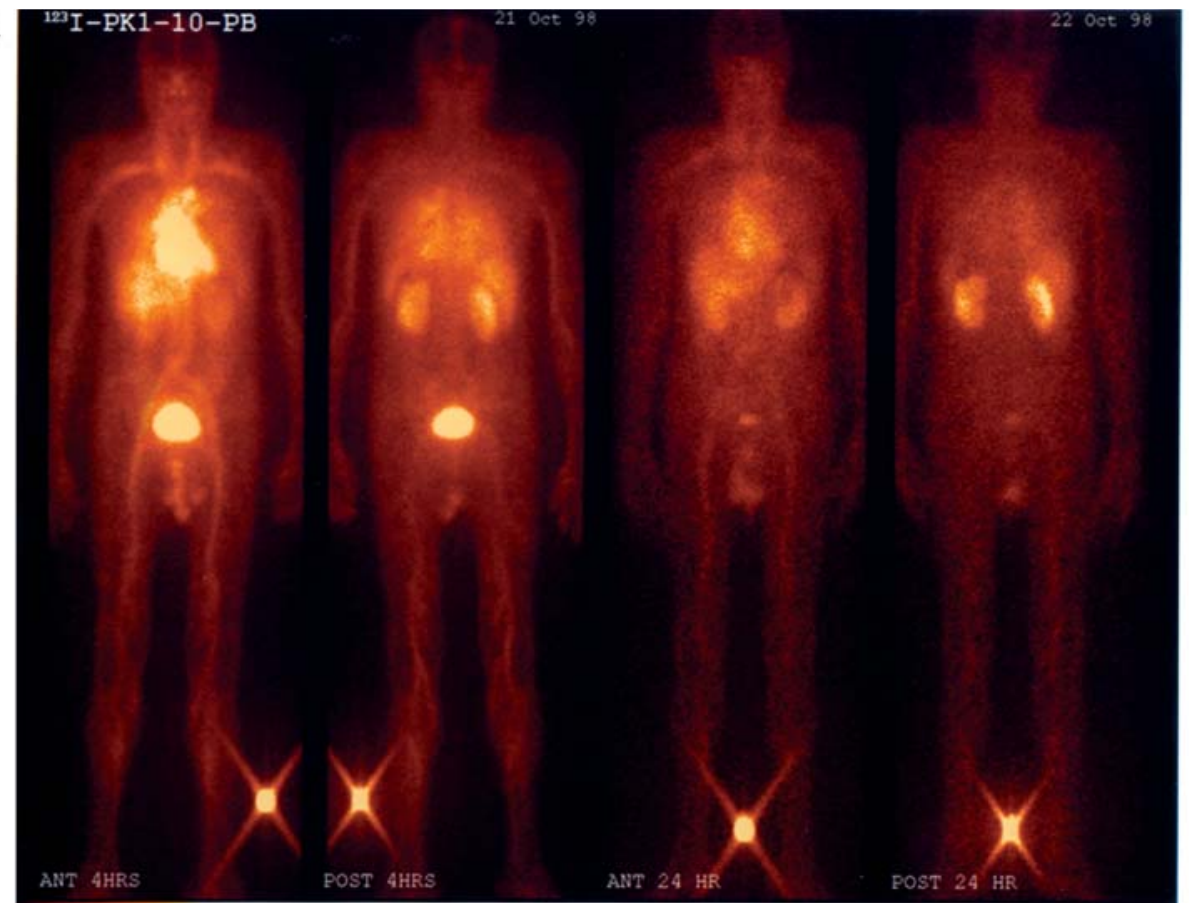

B

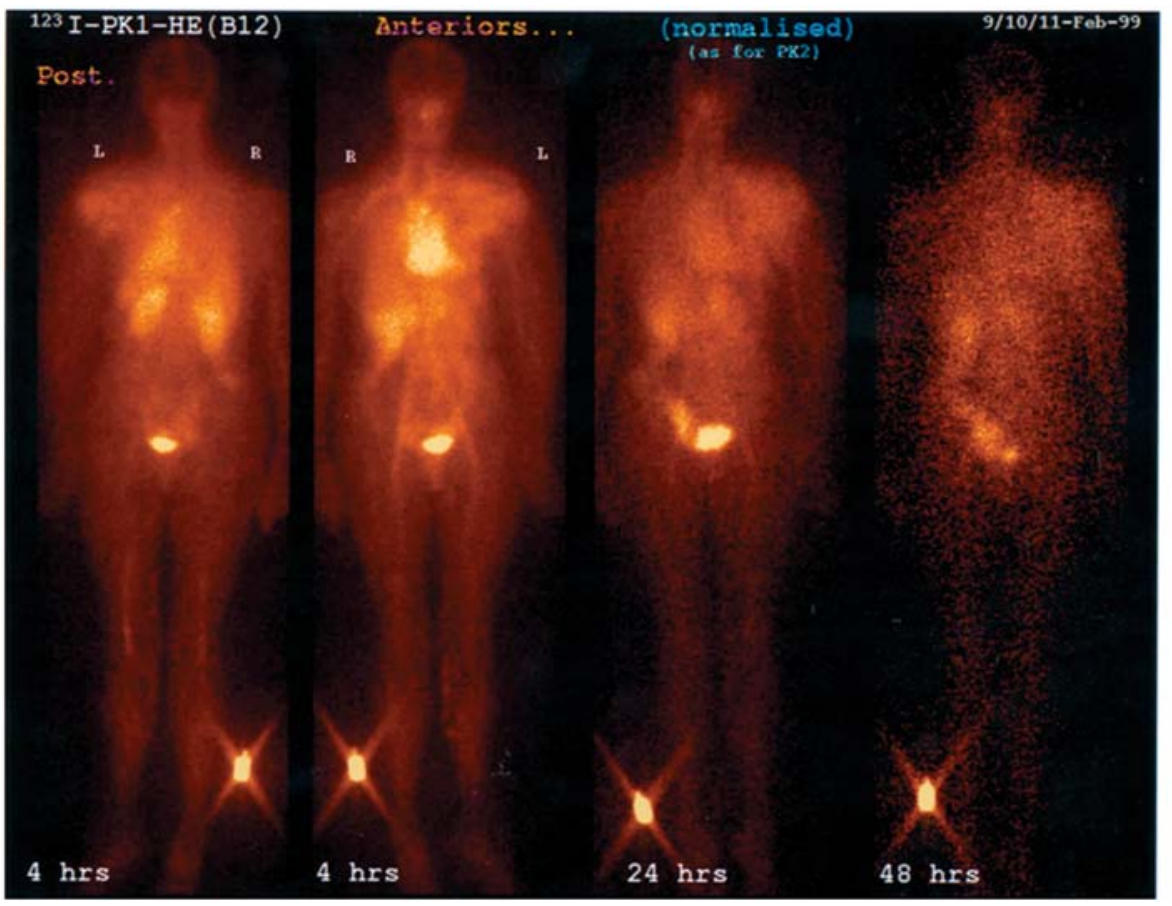

Figure 4. Planar $\gamma$-camera imaging of patients at 4,24 and $48 \mathrm{~h}$ following administration of FCE28068 (280 mg doxorubicin-equivalent $\left./ \mathrm{m}^{2}\right)$. Images were calibrated using an external standard placed between the patient's feet. The patient shown in (A) has large cell lung carcinoma; the patient shown in (B) has metastatic breast cancer with a substantial tumour mass on her left shoulder.

respectively. Although free doxorubicin is known to have minor activity in NSCLC, the drug is not considered active with $4 / 88$ patients responding in the world literature $(16,17)$. It would, therefore, be interesting to study FCE28068 further in patients with NSCLC, to define more clearly its level of activity and its mechanism of action. Moreover, the activity of FCE28068 in NSCLC raises the possibility of applying the same principles of delivery to chemotherapeutic agents with greater intrinsic activity in this disease.
Confirming observations in the phase I study, FCE28068 also showed less toxicity than would be expected from the equivalent dose of free doxorubicin. Most notable was the low rate of alopecia, a particularly significant side-effect of most cytotoxics including conventional anthracyclines and affecting quality of life. FCE28068 also appears less cardiotoxic than the same dose of conventional doxorubicin. Overall, the profile of this drug compares favourably with other experimental therapies. 
The kinetics of blood clearance for the radiolabelled FCE28068 imaging analogue (with plasma distribution and elimination half lives averaging 3.0 and $41.2 \mathrm{~h}$ ) were very similar to those previously reported by analysis of polymerbound doxorubicin (9) (1.8 and $93 \mathrm{~h}$ distribution and elimination half lives, respectively) including by population analysis (distribution and elimination half lives 2.7 and $49 \mathrm{~h}$ respectively) (10). This suggests that the blood kinetics of the radiolabelled imaging agent closely reflect those of the unlabelled polymer conjugate, and reinforces its usefulness as a non-invasive probe to monitor biodistribution and tumour accumulation of the drug.

It is likely that the anticancer activity of doxorubicin delivered as FCE28068 is determined largely by its kinetics and biodistribution. A key difference from free doxorubicin is the extended plasma circulation of the polymer conjugate, an important prerequisite for passive accumulation within tumour tissues. The low volume of distribution and extended bloodstream circulation of the imaging analogue were clearly visible by $\gamma$-camera imaging after $4 \mathrm{~h}$, confirming the results gained by analysis of blood samples. Free doxorubicin has a relatively short plasma half life (18) and the plasma AUC calculated for polymer-bound doxorubicin, assuming the conjugate remains intact (5.0 mM.h), is much greater than is seen for free doxorubicin given at doses of $30-70 \mathrm{mg} / \mathrm{m}^{2}$ [typically 2-10 $\mu$ M.h $(19,20)$ ]. Liposomal doxorubicin also has a much greater AUC than the free drug, measured at 2.9$5.9 \mathrm{mM}$.h over the dose range $35-70 \mathrm{mg} / \mathrm{m}^{2}(21)$, similar to that seen here for the doxorubicin polymer conjugate.

Although the uptake of FCE28068 into tumour masses in this study is hard to quantify from many of the images obtained (largely because of difficulties of image registration for nonsurface tumours), analysis of the patients with axillary breast cancer masses that can be imaged relatively easily suggests accumulation of the administered dose of FCE28068 at levels between 0.7 and $3.0 \%$ greater than in the contralateral shoulder, measured after $24 \mathrm{~h}$. This corresponds to $4-14 \mathrm{mg}$ of doxorubicin. If all the doxorubicin were released from the polymer carrier this could generate doxorubicin levels of 100-300 $\mu \mathrm{M}$ within $100 \mathrm{ml}$ tumour, comparing well with the $0.5-2.0 \mu \mathrm{M}$ of free doxorubicin found in breast cancer biopsies following treatment with conventional doxorubicin (22). The covalent bond between the doxorubicin and polymer carrier is not broken instantly, however, and the real levels of free, active, drug released are likely to be much lower.

In contrast to the hints of activity seen here, a related strategy using a polymer carrier to deliver the topoisomerase I inhibitor camptothecin (PNU166148) recently reported negative results in a phase I and pharmacokinetic study involving 23 patients with various types of cancer (6 colorectal, 4 unknown primary, 2 each head and neck, NSCLC, renals and one of each adrenal, cervical, oesophageal, Ewing's, gastric, mesothelioma and prostate) (23). The linkage of camptothecin to the polymer carrier was designed for $\mathrm{pH}$ catalysed esterolytic cleavage, in contrast to the proteasebased release of doxorubicin employed here, making it hard to compare the pharmacology of the two drug conjugates. Nevertheless, a further study administered PNU166148 to patients with colorectal cancer prior to tumour resection, and found disappointingly low levels of the drug within resected tumour tissue (24). This suggests that the EPR effect does not operate very effectively in clinical colorectal cancer, and this may contribute to the lack of activity seen against colorectal cancer in the present study as well (although the minor response in colorectal cancer observed in the phase I study should not be completely overlooked).

The differential activities of FCE28068 in different cancer types, coupled with apparently differential accumulation of drug conjugates, may relate in part to differences in tumour biology between tumours of different origins. Passive targeting of drug conjugates to tumour tissue (at least in animal models) is thought to be facilitated by enhanced permeability of tumour-associated vasculature, with retention aided by poor or absent lymphatic pathways for drainage of macromolecules from the tumour interstitium. In addition the rate of fluid extravasation will likely depend on the tumour interstitial hydrostatic pressure, which will also be dependent on the efficiency of fluid drainage pathways, giving a complex overall effect. In this context it is noteworthy that whereas clinical lung and breast cancer are recorded as having relatively low interstitial hydrostatic pressures $(10.0 \mathrm{~mm} \mathrm{Hg}, \mathrm{n}=26$, and $15.0 \mathrm{~mm} \mathrm{Hg}, \mathrm{n}=8$, respectively), metastatic colorectal cancer appears to have a substantially higher interstitial pressure (21 mm Hg, n=8) (25). Further evaluation of clinical tumour interstitial pressures and possible correlation with uptake of drug conjugates would appear to be warranted, as this might inform on disease types suitable for treatment with macromolecular drug conjugates.

This macromolecular form of doxorubicin combines decreased toxicity with signs of activity in some types of solid cancer, reinforcing the emerging paradigm for changing the toxicity and efficacy of anticancer agents by forming macromolecular prodrugs with modified biodistribution and activity profiles. This approach may be used in the future with established cytotoxics or to deliver anticancer agents that are otherwise too insoluble (26) or too toxic to be administered in the clinic (4), and conjugates can be designed to release drug with a variety of trigger mechanisms including falling pH (27-29) and reducing environment (30). The polymer carrier also permits incorporation of membrane active peptides or targeting agents into the conjugate structure, the latter designed to enhance receptor-mediated uptake into tumour cells or organs containing tumour metastases, and increase the exposure of the tumour to active drug while minimizing exposure of normal tissues (31). Thoughtful design of macromolecular drug conjugates should yield a range of new approaches to cancer treatment with potentially significant benefits, and several have already progressed as far as clinical evaluation in phase I studies (23,31-33).

\section{Acknowledgements}

We are grateful to Cancer Research UK for financial support and to Pharmacia and Upjohn for supplying the polymeric drugs.

\section{References}

1. Ehrlich P: Chemotherapeutics: scientific principles, methods, and results. Lancet 2: 445-451, 1913. 
2. Maeda H, Seymour LW and Miyamoto Y: Conjugates of anticancer agents and polymers: advantages of macromolecular therapeutics in vivo. Bioconjug Chem 3: 351-362, 1992.

3. Thanou M and Duncan R: Polymer-protein and polymer-drug conjugates in cancer therapy. Curr Opin Investig Drugs 4: 701-709, 2003.

4. Duncan R: The dawning era of polymer therapeutics. Nat Rev Drug Discov 2: 347-360, 2003.

5. Seymour LW: Passive tumor targeting of soluble macromolecules and drug conjugates. Crit Rev Ther Drug Carrier Syst 9: 135-187, 1992.

6. Matsumura $\mathrm{Y}$ and Maeda $\mathrm{H}$ : A new concept for macromolecular therapeutics in cancer chemotherapy: mechanism of tumoritropic accumulation of proteins and the antitumor agent smancs. Cancer Res 46: 6387-6392, 1986.

7. Duncan R: Polymer conjugates for tumour targeting and intracytoplasmic delivery. The EPR effect as a common gateway? Pharm Sci Technolo Today 2: 441-449, 1999.

8. Seymour LW, Ulbrich K, Steyger PS, Brereton M, Subr V, Strohalm J and Duncan R: Tumour tropism and anti-cancer efficacy of polymer-based doxorubicin prodrugs in the treatment of subcutaneous murine B16F10 melanoma. Br J Cancer 70: 636-641, 1994

9. Vasey PA, Kaye SB, Morrison R, Twelves C, Wilson P, Duncan R, Thomson AH, Murray LS, Hilditch TE, Murray T, Burtles S, Fraier D, Frigerio E and Cassidy J: Phase I clinical and pharmacokinetic study of PK1 [N-(2-hydroxypropyl)methacrylamide copolymer doxorubicin]: first member of a new class of chemotherapeutic agents-drug-polymer conjugates. Cancer Research Campaign Phase I/II Committee. Clin Cancer Res 5: 83-94, 1999.

10. Thomson AH, Vasey PA, Murray LS, Cassidy J, Fraier D, Frigerio E and Twelves C: Population pharmacokinetics in phase I drug development: a phase I study of PK1 in patients with solid tumours. Br J Cancer 81: 99-107, 1999.

11. Rejmanova P, Pohl J, Baudys M, Kostka V and Kopecek J: Degradation of oligopeptide sequences in N-(2-hydroxypropyl) methacrylamide copolymers by bovine spleen cathepsin. Makromol Chem B 184: 2009-2016, 1983.

12. Duncan RSL, Flanagan PA, O'Hare KB, Wedge SR, Ulbrich K, Strohalm J, Subr V, Spreafico F, Grandi M, Ripamonti M, Farao $\mathrm{M}$ and Suarato A: Preclinical evaluation of polymerbound doxorubicin. J Controlled Release 19: 331-342, 1992

13. Jones AT, Gumbleton M and Duncan R: Understanding endocytic pathways and intracellular trafficking: a prerequisite for effective design of advanced drug delivery systems. Adv Drug Deliv Rev 55: 1353-1357, 2003.

14. Pimm MV, Perkins AC, Strohalm J, Ulbrich K and Duncan R: Gamma scintigraphy of a 123I-labelled N-(2-hydroxypropyl) methacrylamide copolymer-doxorubicin conjugate containing galactosamine following intravenous administration to nude mice bearing hepatic human colon carcinoma. J Drug Target 3: 385-390, 1996

15. Julyan PJ, Seymour LW, Ferry DR, Daryani S, Boivin CM, Doran J, David M, Anderson D, Christodoulou C, Young AM, Hesslewood S and Kerr DJ: Preliminary clinical study of the distribution of HPMA copolymers bearing doxorubicin and galactosamine. J Control Release 57: 281-290, 1999.

16. Praga $\mathrm{C}$ : Co-operative clinical study of adriamycin in advanced lung tumours. In: International Symposium on Adriamycin. DKaG M (ed). Springer Verlag, Berlin, pp173-179, 1972.

17. Bonadonna G, De Lena M and Beretta G: Preliminary clinical screening with adriamycin in lung cancer. Eur J Cancer 7: 365-367, 1971 .
18. Cummings J and Smyth JF: Pharmacology of adriamycin: the message to the clinician. Eur J Cancer Clin Oncol 24: 579-582, 1988.

19. Conley BA, Egorin MJ, Whitacre MY, Carter DC, Zuhowski EG and van Echo DA: Phase I and pharmacokinetic trial of liposomeencapsulated doxorubicin. Cancer Chemother Pharmacol 33: 107-112, 1993.

20. Richly H, Kupsch P, Passage K, Grubert M, Hilger RA, Kredtke S, Voliotis D, Scheulen ME, Seeber S and Strumberg D: A phase I clinical and pharmacokinetic study of the Raf kinase inhibitor (RKI) BAY 43-9006 administered in combination with doxorubicin in patients with solid tumors. Int J Clin Pharmacol Ther 41: 620-621, 2003.

21. Lyass O, Uziely B, Ben-Yosef R, Tzemach D, Heshing NI, Lotem M, Brufman G and Gabizon A: Correlation of toxicity with pharmacokinetics of pegylated liposomal doxorubicin (Doxil) in metastatic breast carcinoma. Cancer 89: 1037-1047, 2000.

22. Cummings J and McArdle CS: Studies on the in vivo disposition of adriamycin in human tumours which exhibit different responses to the drug. Br J Cancer 53: 835-838, 1986.

23. Schoemaker NE, van Kesteren C, Rosing H, Jansen S, Swart M, Lieverst J, Fraier D, Breda M, Pellizzoni C, Spinelli R, Grazia Porro M, Beijnen JH, Schellens JH and ten Bokkel Huinink WW: A phase I and pharmacokinetic study of MAG-CPT, a watersoluble polymer conjugate of camptothecin. Br J Cancer 87: 608-614, 2002.

24. Sarapa N, Britto MR, Speed W, Jannuzzo M, Breda M, James CA, Porro M, Rocchetti M, Wanders A, Mahteme H and Nygren P: Assessment of normal and tumor tissue uptake of MAG-CPT, a polymer-bound prodrug of camptothecin, in patients undergoing elective surgery for colorectal carcinoma. Cancer Chemother Pharmacol 52: 424-430, 2003.

25. Jain RK: Barriers to drug delivery in solid tumors. Sci Am 271: 58-65, 1994.

26. Vicent MJ, Manzanaro S, De la Fuente JA and Duncan R: HPMA copolymer-1,5-diazaanthraquinone conjugates as novel anticancer therapeutics. J Drug Target 12: 503-515, 2004.

27. Vicent MJ, Tomlinson R, Brocchini S and Duncan R: Polyacetal-diethylstilboestrol: a polymeric drug designed for $\mathrm{pH}$ triggered activation. J Drug Target 12: 491-501, 2004.

28. Ulbrich K, Etrych T, Chytil P, Jelinkova M and Rihova B: Antibody-targeted polymer-doxorubicin conjugates with $\mathrm{pH}$ controlled activation. J Drug Target 12: 477-489, 2004.

29. Pechar M, Braunova A, Ulbrich K, Jelinkova M and Rihova B: Poly(ethylene glycol)-doxorubicin conjugates with $\mathrm{pH}$-controlled activation. J Bioactive Compatible Polymers 20: 319-342, 2005.

30. Saito G, Swanson JA and Lee KD: Drug delivery strategy utilizing conjugation via reversible disulfide linkages: role and site of cellular reducing activities. Adv Drug Deliv Rev 55: 199-215, 2003.

31. Seymour LW, Ferry DR, Anderson D, Hesslewood S, Julyan PJ, Poyner R, Doran J, Young AM, Burtles S and Kerr DJ: Hepatic drug targeting: phase I evaluation of polymer-bound doxorubicin. J Clin Oncol 20: 1668-1676, 2002.

32. Meerum Terwogt JM, Ten Bokkel Huinink WW, Schellens JH, Schot M, Mandjes IA, Zurlo MG, Rocchetti M, Rosing H, Koopman FJ and Beijnen JH: Phase I clinical and pharmacokinetic study of PNU166945, a novel water-soluble polymerconjugated prodrug of paclitaxel. Anticancer Drugs 12: 315-323, 2001.

33. Veronese ML, Flaherty K, Kramer A, Konkle BA, Morgan M, Stevenson JP and O'Dwyer PJ: Phase I study of the novel taxane CT-2103 in patients with advanced solid tumors. Cancer Chemother Pharmacol 55: 497-501, 2005. 